\title{
Exotic muon decays and the KARMEN anomaly
}

\author{
S.N. Gninenko円 and N.V. Krasnikovt \\ Institute for Nuclear Research of the Russian Academy of Sciences, Moscow \\ 117312
}

\begin{abstract}
An anomaly in time distribution of neutrinos from the ISIS pulsed beam stop source observed by the KARMEN collaboration is discussed. We show that the anomaly can be interpreted as a superposition of two exponentials, both having time constants consistent with the $\mu^{+}$lifetime of $2.2 \mu \mathrm{s}$. It is assumed that they both originate from muon decays at rest. One of them describes the time distribution of the prompt neutrino events, while the other describes the time distribution of events from delayed decays of slowly moving $(\beta \simeq 0.02)$ particles in the KARMEN calorimeter.

We propose here that these particles are produced in exotic decays of positive muons $\mu^{+} \rightarrow e^{+}+X$, resulting in the second exponential time distribution shifted by the time of flight with respect to the time distribution of neutrino events. This model gives an aceptable fit to the KARMEN data if $X$ has a mass of $103.9 \mathrm{MeV}$. The possible decay modes of this new massive neutral particle are discussed. This hypothesis can be experimentally tested in the near future by studying the low energy part of the $e^{+}$ spectrum in the $\mu^{+}$decays.
\end{abstract}

\section{Introduction}

The KARMEN collaboration, which studies the interactions of neutrinos from the stopped $\pi^{+}$decay chain at Rutherford Appelton Laboratory, has reported an anomaly [1] in the time distribution of neutrino events in the KARMEN calorimeter. There is a bump of events in the distribution which was expected to be well described by a single exponential with a time constant equal to the muon decay lifetime.

It was suggested that a new weakly - interacting half spin particle with a mass $33.9 \mathrm{MeV} / \mathrm{c}^{2}$ from initial $\pi^{+} \rightarrow \mu^{+}+x$ decay, which travels with velocity $\beta=v / c \simeq 1 / 60$ and decays in the KARMEN calorimeter after a mean flight path of 17.5 metres, can be a possible candidate for an explanation of this anomaly. Since the visible energy in the calorimeter was in the range from 11 to $35 \mathrm{MeV}$ it was also assumed that these new particles deposited only a fraction of their

\footnotetext{
${ }^{1}$ E-mail address: Sergei.Gninenko@cern.ch

${ }^{2}$ E-mail address: nkrasnik@vxcern.cern.ch
} 
full energy in the calorimeter. The hypothetical $\pi^{+} \rightarrow \mu^{+}+x$ decay has been searched for at PSI by studying muons from pion decay in flight [2]. The result gives a branching ratio of less than $7 \times 10^{-8}$ at $95 \%$ Confidence Level (C.L.) for this decay mode leaving only a narrow allowed region in the branching ratio if combined with the results of ref. [3].

Another possible interpretation of the anomaly proposed by the KARMEN collaboration is related to the direct production of a new particle in the proton collision with the neutrino target. However, the collaboration has not yet stated whether it has devised one.

Concerning the possible explanation of the anomaly by exotic muon decays it was concluded that "...the narrow clustering of the anomalous events rules out contributions from $\mu^{+}$decay, which spreads over a much longer time interval (compared to the $\pi^{+}$- life time of $\approx 20 \mathrm{~ns}$ ) up to $10 \mu \mathrm{s}$ after beam-on target." [回].

This conclusion is the motivation for present letter, where we show that the KARMEN anomaly could be explained by contributions from the exotic $\mu^{+}$decays.

The paper is organised as follows. In section 2 we analyse the time distribution obtained by the KARMEN collaboration and give a possible explanation of the anomaly. As an example we consider the $\mu^{+} \rightarrow e^{+}+X$ decay into a positron and a new boson with the mass of $103.9 \mathrm{MeV} / \mathrm{c}^{2}$. In sections 3,4 the possible X-boson phenomenologies and experiments on searching for the rare decay $\mu^{+} \rightarrow e^{+}+X$ are discussed, respectively. Section 5 contains concluding remarks.

\section{Analysis of the neutrino time spectrum}

Figure 1 shows the time distribution found by KARMEN with respect to beamon target events. Analysis of this time distribution was performed in ref. [1] after subtraction of the cosmic ray induced background, which was found to be constant and equal to 60 events per $0.5 \mu s$.

This spectrum was fitted in ref. [1] by a superposition of Gaussian and exponential signals from muon decay neutrinos having a $2.2 \mu \mathrm{s}$ decay constant. The fit procedure performed with the minimization routines of MINUIT [4] results in $\chi^{2}$ of 9.7 for 14 degrees of freedom (DF) corresponding to $79 \%$ C.L. for this hypothesis. Gaussian, which is centred at $(3.6 \pm 0.25) \mu s$ with a full width at half maximum(FWHM) of $(1.3 \pm 0.5) \mu s$ consisted of $83 \pm 28$ events associated with the signals from decay in flight of the proposed particle in the fiducial volume of the KARMEN calorimeter.

We tried to reproduce these results by using the same MINUIT minimizing

\footnotetext{
${ }^{3}$ The experimental points were reproduced from the original KARMEN publication [1]. The error bars were calculated taking into account the errors due to subtraction of the background.
} 
routines. Our results and results of the KARMEN fit were found to be in a good agreement: our fit procedure yielded $\chi^{2}$ of 9.8 for 14 degrees of freedom (DF) corresponding to $77 \%$ C.L. for this hypothesis. Gaussian was found to be centred at $(3.7 \pm 0.26) \mu s$ with a FWHM of $(1.3 \pm 0.5) \mu s$ and with $85 \pm 32$ events. The result of our fit procedure is shown in Figure 1.

Having this good agreement in mind, we then performed an exponential fit of the KARMEN time distribution assuming that this spectrum is described by a sum of two exponentials: the first exponential describes the time distribution in the interval $1-3.3 \mu \mathrm{s}$, and the second one describes the time distribution in the interval $3.3-10 \mu \mathrm{s}$. Figure 2 shows the result of the fit (solid lines). The MINUIT minimizing routines yield a $\chi^{2}$ of 9.7 for 15 DF corresponding to $84 \%$ C.L., and time constants of $(2.29 \pm 0.34) \mu s$ and $(2.1 \pm 0.6) \mu s$ for the first and the second exponentials respectively. Note that there is no significant difference in Confidence Levels of our and KARMEN's hypotheses.

The time width of the transition region between two exponentials is rather small and corresponds to $\simeq 0.5 \mu \mathrm{s}$. We try to fit this region more precisely using a Gaussian shape of the transition curve instead of a step-function. However, no significant difference between two results has been found.

A similar description of the time distribution was obtained by superimposing an exponential describing the distribution of events in the time interval 3.3 $10 \mu s$ upon an exponential signal having a $2.2 \mu s$ decay constant for the full time interval. Figure 3 shows the corresponding time distribution of events after subtraction of the exponential part with $2.2 \mu \mathrm{s}$. A direct exponential fit of the time distribution of events in this region gives a time constant of $2.26 \mu s$ close to the value of muon decay constant; however the error is rather large because of poor statistics.

Since the time constants were found to be equal within the error limits to the muon decay constant we may assume that all events in the KARMEN time distribution originate from the muon decays.

Thus, our interpretation of the KARMEN anomaly is the following. Positive muons from $\pi^{+}$decays stop in the target ( muon range in the target is $\simeq 1 \mathrm{~cm}$ ) and decay giving a flux of neutrinos which decreases in time exponentially with the muon decay constant. The first exponential shown in the time spectrum in Figure 2 is related to the interactions of this flux of neutrinos in the KARMEN calorimeter. An excess of events after $\simeq 3.6 \mu \mathrm{s}$ shown in Figure 3 is related to the decays of slow moving particles produced in muon decays. Therefore, the flux of these particles and fraction of their decays in the fiducial volume of the KARMEN calorimeter also decrease in time exponentially with the muon decay constant, resulting in the second time distribution but shifted by the time of flight with respect to the time distribution of neutrino events. Since the time width of the transition region between two exponentials is rather narrow (see Figure 2), we suggest that these new particles originate from the two-body decays of positive 
muons, namely $\mu \rightarrow e^{+}+X$. We note that the RMS of fluctuations of time needed for $X$ bosons to pass from the neutrino target to the calorimeter and decay inside its volume was estimated to be of the order of $0.2 \mu \mathrm{s}$. The effect of the convolution of these fluctuations and the exponential from muon decay on the exponential shape of this curve and its decay constant was found to be small.

The mass of the $X$ boson calculated for $\beta_{X}=1 / 60$ is found to be $(103.9 \pm 0.1)$ $\mathrm{MeV} / \mathrm{c}^{2}$, where the error corresponds to the spread of $X$ 's velocity estimated from the time width of the transition region between two exponentials. The excess of events after subtraction of the contribution from the neutrino was found to be $\Delta N=108 \pm 41$ events.

The limit on branching ratio $B r=\Gamma\left(\mu \rightarrow e^{+}+X\right) / \Gamma(\mu \rightarrow$ all $)$ as a function of the $X$-boson lifetime can be recalculated from the KARMEN result for $\pi^{+} \rightarrow$ $\mu^{+}+x$ decay mode taking into account the difference in the number of excess events found after the fit procedure. The result is shown in Figure 4.

\section{Possible X-boson phenomenologies}

Suppose that X-boson is a neutral scalar particle. The Lagrangian describing the $\mu \rightarrow e+X$ decay has the form

$$
L=X\left[h_{\mu e L} \bar{\mu}_{L} e_{R}+h_{\mu e R} \bar{\mu}_{R} e_{L}+\text { h.c. }\right]
$$

The $\mu \rightarrow e+X$ decay width is given by the formula

$$
\Gamma(\mu \rightarrow e+X)=\frac{m_{\mu}}{32 \pi}\left(h_{\mu e L}^{2}+h_{\mu e R}^{2}\right)\left(1-\frac{m_{X}^{2}-m_{e}^{2}}{m_{\mu}^{2}}\right) \cdot\left[\left(1+\frac{m_{e}^{2}-m_{X}^{2}}{m_{\mu}^{2}}\right)^{2}-4 \frac{m_{e}^{2}}{m_{\mu}^{2}}\right]^{\frac{1}{2}}
$$

For a non-relativistic X-boson with a speed $v_{X}=\frac{1}{60} c$ we find that its mass is $m_{X}=103.9 \mathrm{MeV}$ and the $\mu \rightarrow e+X$ branching ratio is

$$
\operatorname{Br}(\mu \rightarrow e X) \approx 0.4 \cdot 10^{13}\left[h_{\mu e L}^{2}+h_{\mu e R}^{2}\right]
$$

For the interaction (1) the X-boson decays via the virtual $\mu$ meson $X \rightarrow\left(\mu^{*} \rightarrow\right.$ $e \nu \bar{\nu})+e$ and its decay width is

$$
\Gamma\left(X \rightarrow e^{+} e^{-} \nu \bar{\nu}\right) \sim O\left(\left(\pi^{2} \Gamma(\mu \rightarrow e \nu \bar{\nu})\left(h_{\mu e L}^{2}+h_{\mu e R}^{2}\right)\right)\right.
$$

As it follows from the observed number of the anomalous events due to the $\mathrm{X}$-boson decay in the KARMEN experiment, the ratio

$$
K \equiv B r(\mu \rightarrow e+X) \Gamma(X \rightarrow v i s)
$$

is

$$
K \sim O\left(3 \cdot 10^{-11} s^{-1}\right)
$$


From the formulae $(2-6)$ we find that $\operatorname{Br}(\mu \rightarrow e+X) \sim O\left(10^{-2}\right)$ and $\tau(X \rightarrow$ $\mu e \nu \bar{\nu}) \sim O\left(10^{9}\right) s$, that is probably at the level of the contradiction with the experimental bound [6] $\operatorname{Br}(\mu \rightarrow e+X) \leq 3 \cdot 10^{-4}$ which is valid however for $m_{X} \geq 103.5 \mathrm{MeV}$ (see Section 4). It is possible to invent a model where the $\operatorname{Br}(\mu \rightarrow e+X)$ is less than $O\left(10^{-4}\right)$. Let us introduce the hypothetical heavy charged lepton $\mathrm{L}$ and consider the decay of the $\mathrm{X}$-boson through the virtual charged L-lepton

$$
X \rightarrow\left(L^{*} \rightarrow e \nu \bar{\nu}\right)+e
$$

For the L-lepton mass $m_{L}=100 \mathrm{GeV}$, the coupling constant of L-lepton with electron and X-boson $h_{L e X}=0.1$, the L-lepton mixing with electron $\epsilon_{e L}^{2}=10^{-2}$, we find that $\operatorname{Br}(\mu \rightarrow e X)=O\left(10^{-5}\right)$. So, if our interpretation of the KARMEN anomaly is correct we expect a rather large branching ratio $\operatorname{Br}(\mu \rightarrow e+X) \geq$ $O\left(10^{-5}\right)$. Therefore, it would be very interesting to perform an experiment on the search for $\mu \rightarrow e+X$ decay with improved sensitivity. These scenarios predict the X-boson lifetime $\tau(X) \geq O\left(10^{6}\right)$ s that probably leads to problems with astrophysical and cosmological constraints. Moreover, these scenarios predict an anomalously high X-boson decay energy $<E_{v i s}>\sim 50 \mathrm{MeV}$ compared to the observed visible energy $E_{v i s} \sim(11-35) \mathrm{MeV}$.

To avoid these problems, let us consider the model with two additional neutral scalar bosons $\phi_{1}$ and $\phi_{2}$ and suppose that the $\phi_{1}$-boson is lighter than the X-boson and the $\phi_{2}$-boson is heavier than the $\mathrm{X}$-boson. The Lagrangian describing the $\mathrm{X}$-boson decay has the form

$$
L=\lambda X \phi_{1} \phi_{2}+h_{e} \phi_{2} \bar{e} e+h_{\nu} \phi_{1} \bar{\nu} \nu
$$

The X-boson decays via the reaction

$$
X \rightarrow \phi_{1}+\left(\phi_{2}^{*} \rightarrow e^{+} e^{-}\right)
$$

through the virtual $\phi_{2}$-boson and the neutral $\phi_{1}$-boson decays into neutrino antineutrino pair. For such a scenario it is possible to have $<T_{v i s}>\sim 30 \mathrm{MeV}$ and $\tau(X) \leq 10^{-2} \mathrm{~s}$. For instance, for $m_{X}=103.9 \mathrm{MeV}, m_{\phi_{1}}=73 \mathrm{MeV}, m_{\phi_{2}}=1$ $\mathrm{GeV}, h_{e}=10^{-3}, \lambda=10^{-2} m_{\mu}$ and $h_{\bar{\nu}}=10^{-6}$ we find that the average visible energy in the X-decay is $\left\langle T_{\text {vis }}>\approx 26 \mathrm{MeV}\right.$ and $\tau(X) \sim 3 \cdot 10^{-3} \mathrm{~s}, \tau\left(\phi_{1}\right) \sim 2 \cdot 10^{-10}$ $\mathrm{s}, \operatorname{Br}(\mu \rightarrow e+X) \approx 10^{-13}, \tau\left(\phi_{2}\right) \approx 3 \cdot 10^{-17} \mathrm{~s}$. So in this rather artificial model all particles are not long-lived and it is possible to have $\left\langle T_{v i s}\right\rangle$ in agreement with KARMEN data.

Note also that it is possible to have a model where the $\phi_{2}$-boson is the Higgs boson. For instance, for the Higgs boson mass $m_{h}=100 \mathrm{GeV}$ and $\lambda=m_{\mu}$ we find that $\tau\left(X \rightarrow \phi_{1} e^{+} e^{-}\right) \sim 0.7 \cdot 10^{7} \mathrm{~s}$ and $\operatorname{Br}(\mu \rightarrow e X) \sim 2 \cdot 10^{-4}$. In this case to have $\tau(X) \leq 10^{-2}$ s we have to postulate an additional interaction of the $X$-boson with neutrinos. 


\section{Experimental search for the $\mu^{+} \rightarrow e^{+}+X$ decay}

Since $X$ 's are weakly-interacting and relatively long lived particles, their flux would penetrate any type of calorimeter without significant attenuation and cannot be observed effectively in the detector via its decays. A more effective experimental signature for $\mu^{+} \rightarrow e^{+}+X$ decays would be a peak from mono-energetic positrons in the low energy part of the positron spectrum corresponding to the principal mode of $\mu^{+}$decays.

Direct experimental searches for a peak from $\mu^{+} \rightarrow e^{+}+X$ decays were performed in a few experiments [5]. For the lowest positron energy region from 1.6 to $6.8 \mathrm{MeV}$, corresponding to the mass region of $103.5-98.3 \mathrm{MeV} / \mathrm{c}^{2}$, the branching ratio limit of $\Gamma\left(\mu^{+} \rightarrow e^{+}+X\right) / \Gamma\left(\mu^{+} \rightarrow\right.$ all $) \leq 3 \times 10^{-4}$ was obtained in ref. [6] from the analysis of published data on muon decays in the hydrogen bubble chamber obtained by Derenzo [7]. The result is limited by a poor $(\simeq$ $30-40 \%$ ) momentum resolution in this region.

It would be interesting to search for a peak in the low energy part of the positron spectrum by using, for example, a high resolution Ge-detector ( $\mathrm{NaI}$ detector) with a typical energy resolution of the order of $\mathrm{KeV}(\simeq 100 \mathrm{KeV})$. The low energy muons could be stopped in the active volume of the detector. In this case the low energy spectrum of the positrons could be measured without significant distortions.

The estimate of the rate from the principal muon decays shows that, using Ge-detector, one could expect a sensitivity to the $\mu^{+} \rightarrow e^{+}+X$ decay branching ratio of better than $10^{-6}$. Here it is assumed that the intensity of stops is of the order of $10^{4} \mu^{+} / \mathrm{s}$ and that the exposure to the muon beam is $\simeq 1$ month.

\section{Conclusion}

The anomalous time distribution of the events from the KARMEN detector can be acceptably described by a superposition of the time distribution of neutrino signals following the expected $2.2 \mu s$ muon decay constant and a weaker exponential signal from delayed decays of the new massive neutral particles also originated from the muon decay at rest and shifted in time due to their time of flight.

It is proposed that a new boson $X$ with the mass of $103.9 \mathrm{MeV} / \mathrm{c}^{2}$ which might be produced in the decays of positive muons $\mu^{+} \rightarrow e^{+}+X$ could be a possible candidate for an explanation of the anomaly. The visible energy from the possible decay modes of $X$ 's can be within the allowed region from 11 to 35 $\mathrm{MeV} / \mathrm{c}^{2}$. This hypothesis can be experimentally tested in the near future at a level of sensitivity of better than $10^{-6}$.

It should be noted that the original interpretation of the KARMEN anomaly as a new neutral fermion $x$ produced in $\pi^{+} \rightarrow \mu^{+} x$ decay with a mass $m_{x}=33.9$ $\mathrm{MeV}$ has been discussed in ref. [3]. It has been shown that a mainly sterile 
neutrino scenario is compatible with all laboratory constraints, although there are problems with astrophysical and cosmological constraints. Our interpretation with the scalar $X$-boson produced in the $\mu^{+} \rightarrow e^{+} X$ decay is in the spirit of the familon scenario [8].

\section{Acknowledgements}

We thank Prof. V.Matveev for useful discussions and remarks, Prof. F.Wilczek for useful comment and A.Toropin for help in calculations. One of the authors (S.N.G.) would like to thank his colleagues from the NOMAD collaboration at CERN for fruitful discussions. 


\section{References}

[1] KARMEN Collaboration: B. Armbruster et al., Phys. Lett. B 348(1995)19. K. Eitel, Talk given at CERN on February 17, 1998, where reality of the anomaly was further confirmed by still more such events from the KARMEN detector.

[2] R. Bilger at el., Phys.Lett.B363(1995)41.

[3] V. Barger, R.J.N. Phillips and S. Sarkar, Phys.Lett.B352(1995)365.

[4] F. James and M. Roos, CERNLIB Write Up No. D506, 1977.

[5] Particle Data Group, Phys. Rev. v. D54(1996) part 1.

[6] D.A. Bryman and E.T.H. Clifford, Phys. Rev. Lett.B246(1989)287.

[7] S.E. Derenzo, Phys. Rev. 181 (1969) 1854.

[8] F. Wilczek, Phys.Rev.Lett.49(1982)1549. 


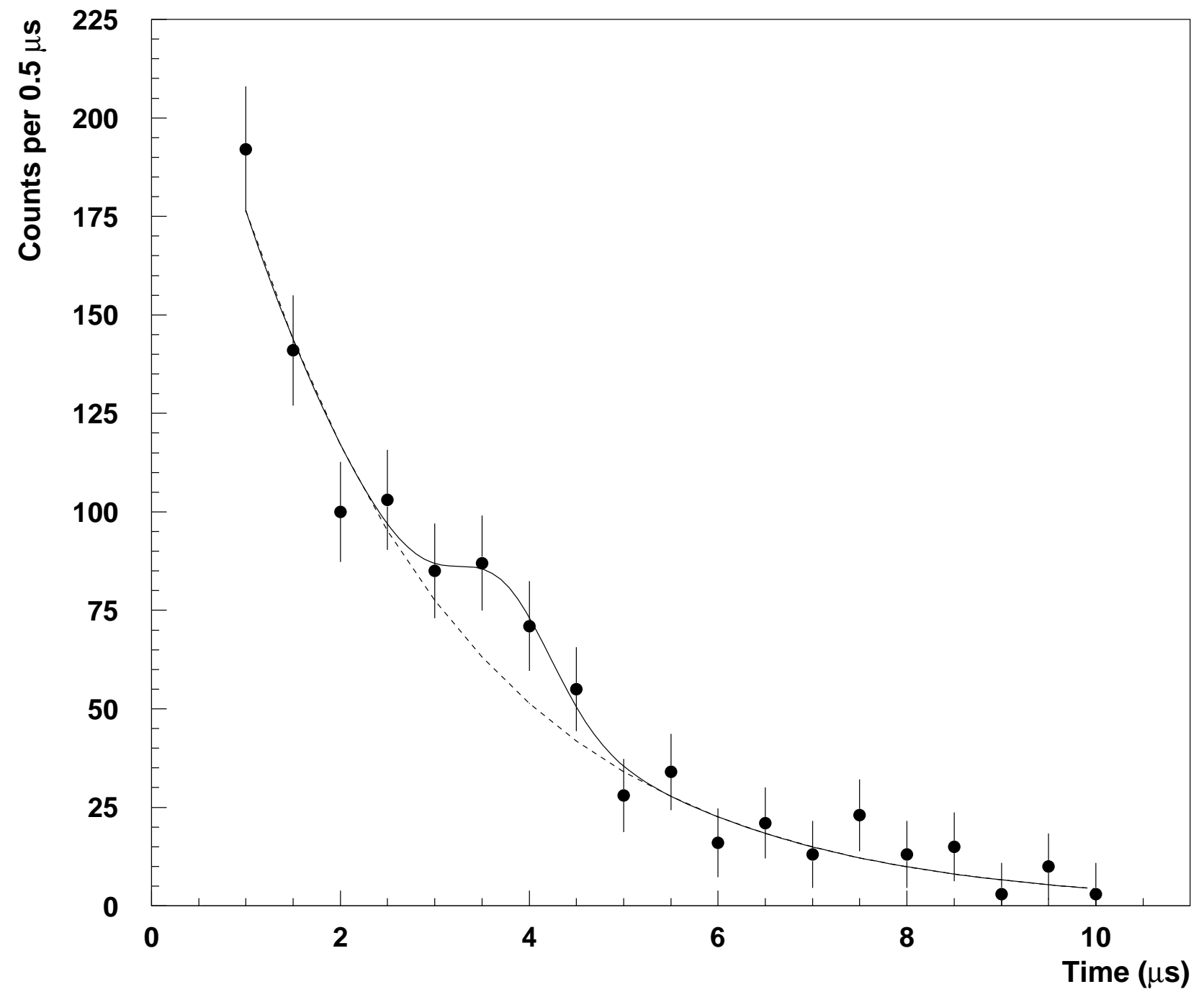

Figure 1: Time distribution of events in the KARMEN calorimeter after the subtraction of the cosmic background. ${ }^{3}$ The data are fitted to an exponential with the 2.2 $\mu$ s decay constant on which is superimposed a Gaussian signal centred at $3.7 \mu \mathrm{s}$. The fit procedure results in $\chi^{2}$ of 9.8 for 14 degrees of freedom. 


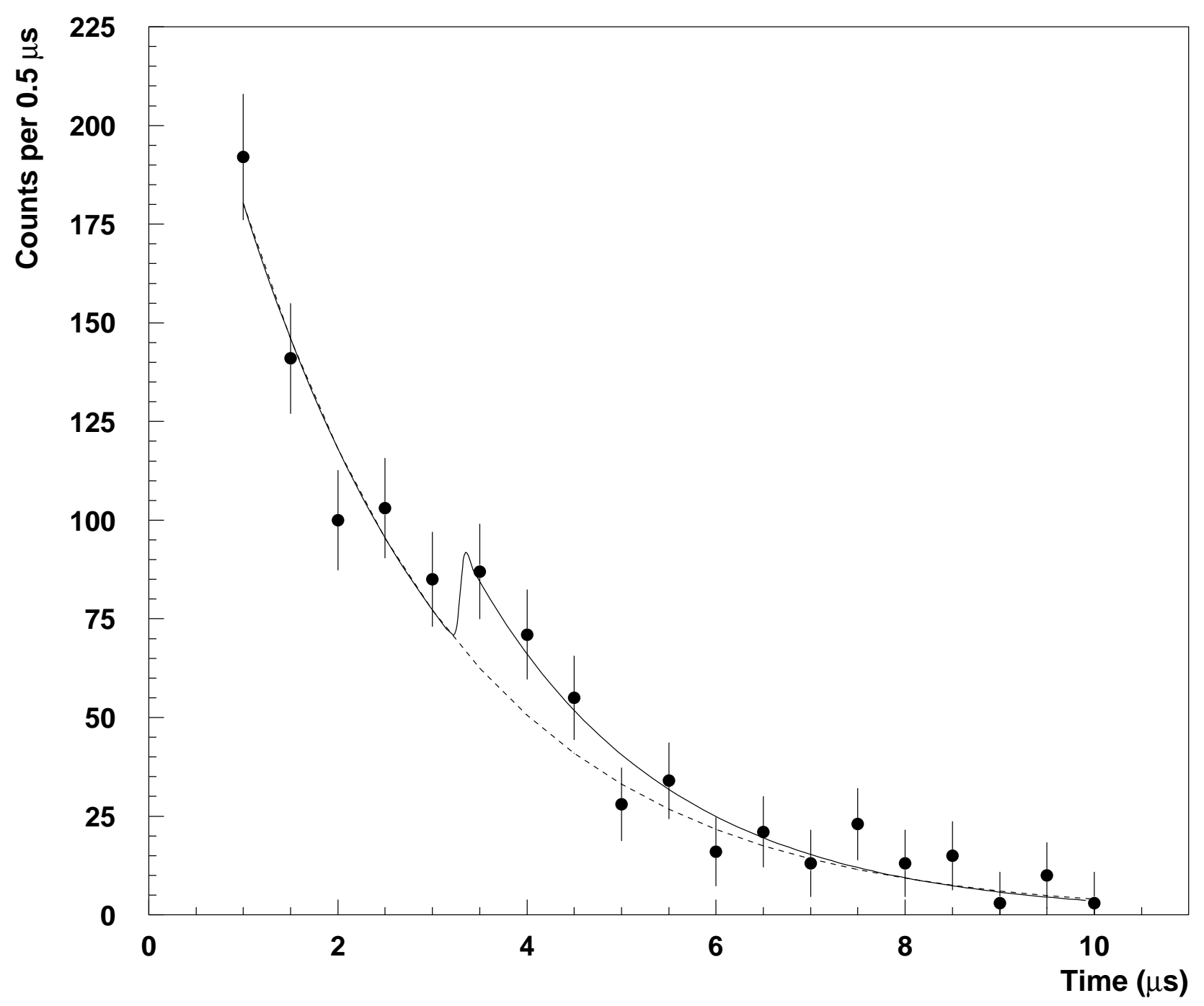

Figure 2: Time distribution of events in the KARMEN calorimeter after the subtraction of the cosmic background. ${ }^{3}$ The solid curves are a fit to the points by a sum of two exponentials. The first exponential describes the time distribution in the region from 1.0 to $3.3 \mu \mathrm{s}$ and the other in the region from 3.3 to $10 \mu \mathrm{s}$ with time constants of $(2.29 \pm 0.34) \mu s$ and $(2.1 \pm 0.6) \mu s$, respectively. The broken line corresponds to the extrapolation of the first exponential. The fit procedure results in $\chi^{2}$ of 9.7 for 15 degrees of freedom. 


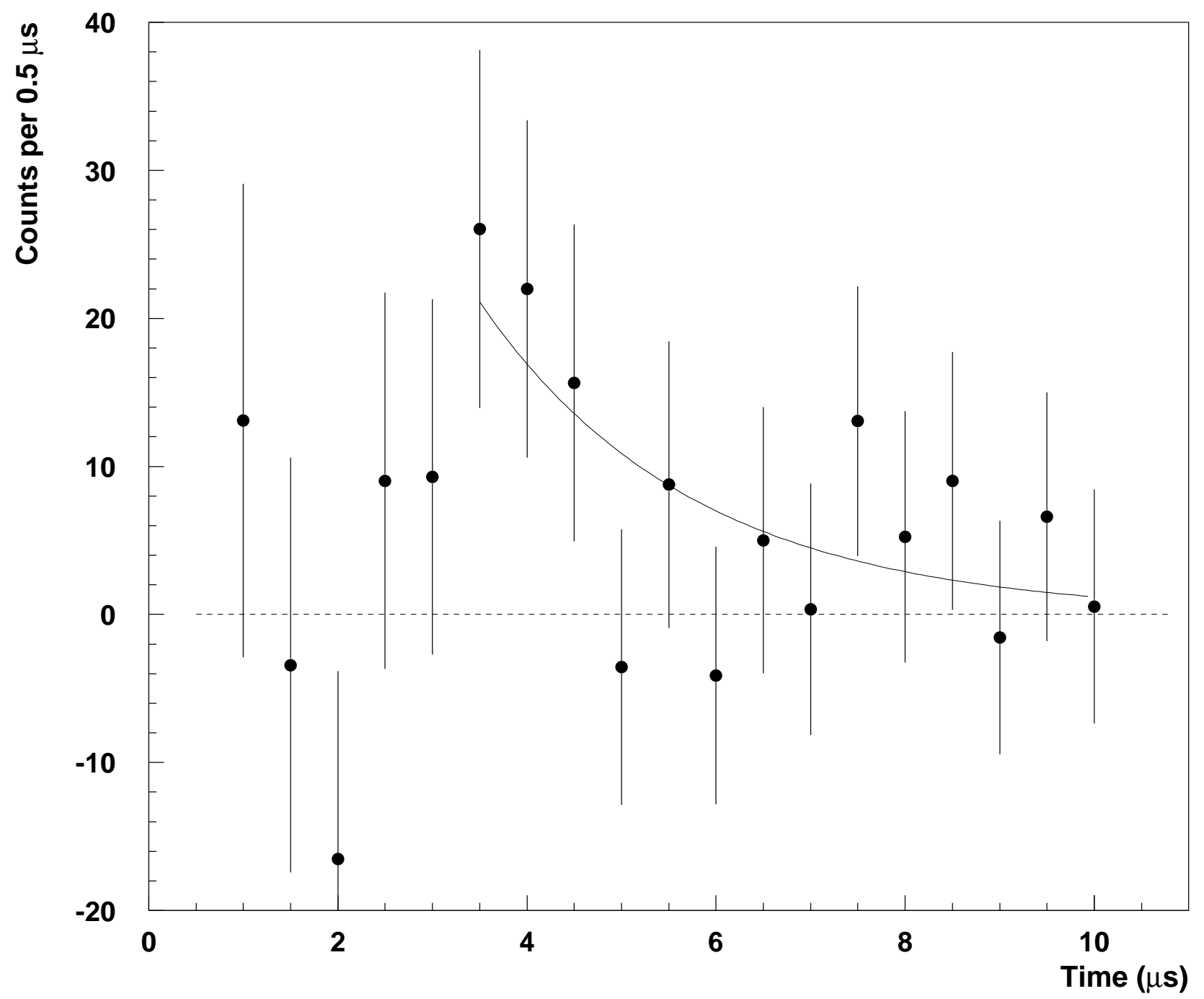

Figure 3: Time distribution of excess events left after subtraction of the exponential component with $2.2 \mu \mathrm{s}$ decay constant. The solid line is the exponential fit to the points in the interval 3.3-10 $\mu$ s which gives a time constant of $2.26 \mu \mathrm{s}$. 


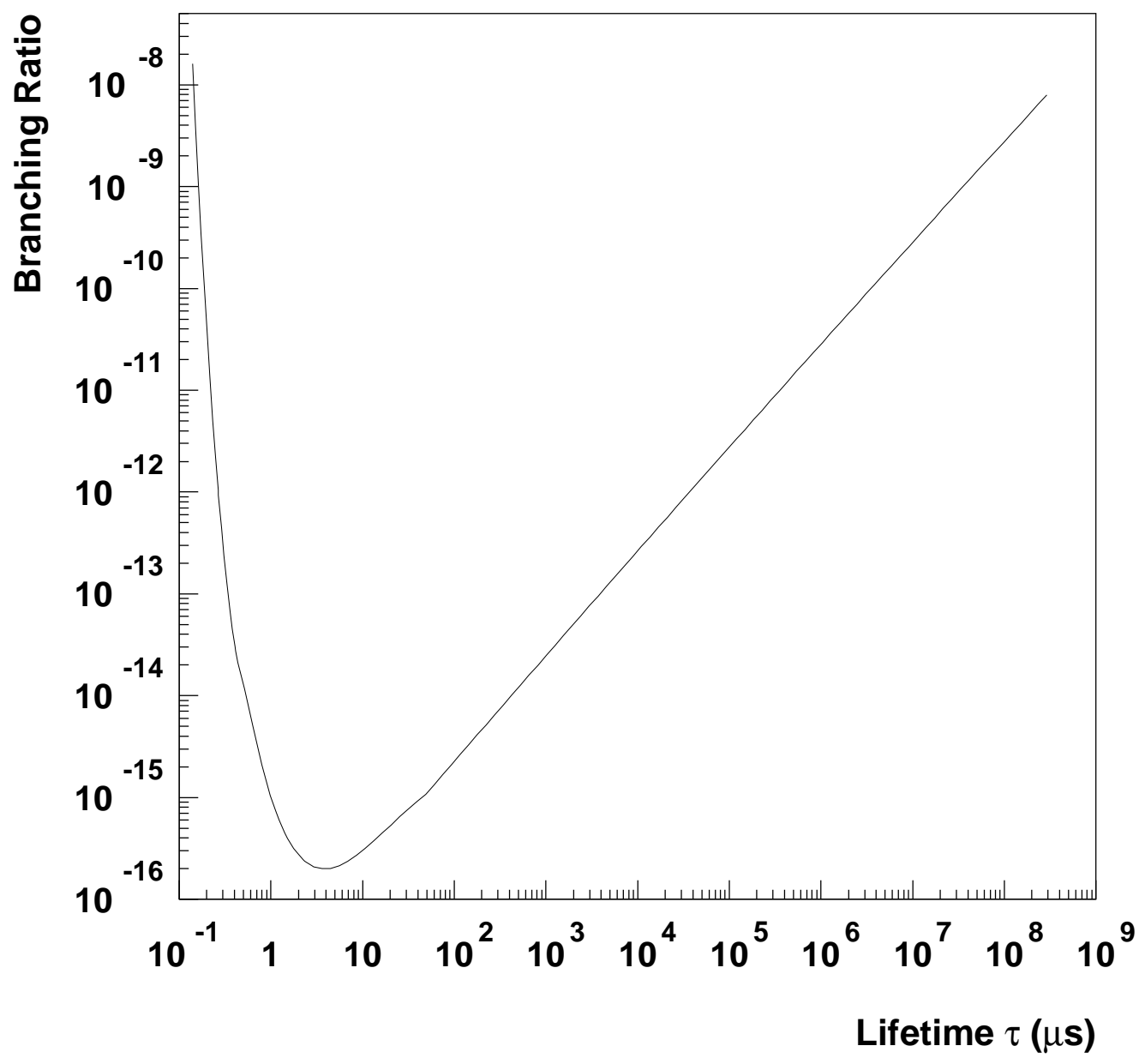

Figure 4: The branching ratio $\Gamma\left(\mu^{+} \rightarrow e^{+}+X\right) / \Gamma\left(\mu^{+} \rightarrow\right.$ all $)$ as a function of the lifetime of the $X$-boson calculated from the number of observed events $(\Delta N=91$ events) using the analogous KARMEN plot for a decay mode of $\pi^{+} \rightarrow \mu^{+}+x$. 